\title{
Business Models of E-Government: Research on Dynamic E-Government Based on Web Services
}

\author{
Li Yan ${ }^{1,2}$, Jiumin Yang ${ }^{3}$ \\ 1 Department of Information Management, Huazhong Normal University, \\ 2 Engineering Research Center of Education Information Technology, \\ Huazhong Normal University, Wuhan, China 430079 \\ yanli@mail.ccnu.edu.cn \\ 3 Department of Information Technology, Huazhong Normal University, \\ Wuhan, China 430079 yjm@mail.ccnu.edu.cn
}

\begin{abstract}
Government transcends all sectors in a society. It provides not only the legal, political and economic infrastructure to support other sectors, but also exerts significant influence on the social factors that contribute to their development. With its maturity of technologies and management, e-government will eventually enter into the time of 'one-stop' services. Among others, the technology of Web services is the major contributor to this achievement. Web services provides a new way of standard-based software technology, letting programmers combine existing computer system in new ways over the Internet within one business or across many, and would thereby bring about profound and far-reaching impacts on e-government. This paper introduced the business modes of egovernment, architecture of dynamic e-government and its key technologies. Finally future prospect of dynamic e-government was also briefly discussed.
\end{abstract}

\section{Introduction}

In the past several years, improvement and modernization of services demanded by the public, the need for increased resource efficiency and the supply of advanced information and communication technologies have formed major agencies to drive contemporary governments to transform to electronic government (e-government). The e-government initially defined as the delivery and administration of government products and services over an information technology infrastructure, aiming at efficiency savings and a more effective administration and seeking to achieve an ecommerce styled public sector model. After that, more people realized the need for

Please use the following format when ciling ihis chapler:

Yan, Li., Yang, J., 2007, in IFIP International Federation for Information Processing, Volume 252, Integration and Innovation Orient to F-Society Volume 2, eds. Wang, W., (Boston: Springer), pp. 421-428. 
greater citizen participation in government. Since then, the democracy element was introduced into the concept of e-government, whereas the impacts of governance and government itself are equally emphasized. The informatization practice of countries all over the world has shown that the level of a government's informatization is one main factor that can affect its international competitive power [1]. Therefore, based on its maturity levels and transformation processes, e-government can be generally divided into three stages: information, transaction and transformation [2].

\section{Web services architecture and technologies}

\subsection{Web services architecture}

A typical web services architecture consists of three entities: service providers, service brokers and service requesters. They provide the three fundamental operations - publish, find and bind - respectively [3].

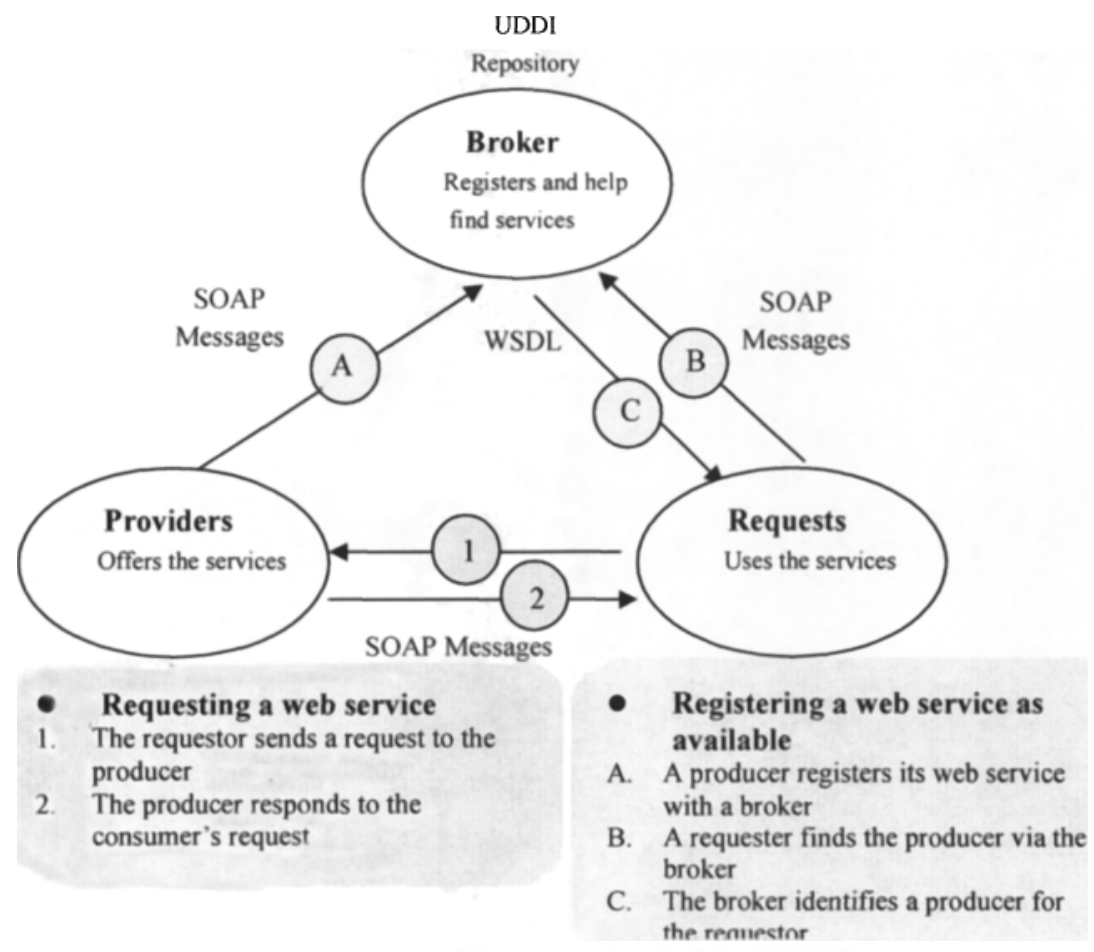

Fig.1. Interaction between service brokers, providers and requests 
- Service providers create web services and publish them by registering the services with service brokers.

- Service brokers maintain a registry of published services.

- Service requesters find required services by searching the service broker's registry, and bind their applications to the service provider to use particular services.

The interaction between these three entities is shown in Figure 1.

\subsection{Web services Technologies}

Web Services Description Language (WSDL), Universal Description, Discovery and Integration (UDDI) and the Simple Object Access Protocol (SOAP) form the core technologies of web services. The roles of each technology in web services will be illustrated in turn $[3,4]$.

\section{- WSDL}

The WSCL is an XML language used to describe the web services interfaces that are accessible from a wide variety of platforms and programming languages. The description includes such details as data type definitions, the operations supported by the services, input/output message formats, network address and protocol bindings. This means WSDL not only describes message contents, but also defines where the service is available and what communication protocol is used to talk to the service. In other words, WSDL file defines everything required to write a program to work with web services.

- UDDI

The UDDI specifies a mechanism to register and locate web services. It lets WS register their characteristics with a registry so that other applications can discover and integrate with them. Users can search for a company that offers the services needed, read about the services offered and contact the company for further information.

In web services, the description of a business and the services it offers are presented by UDDI directory entry. Typically an entry in the UDDI consists of three parts. The 'Yellow Pages' describes the companies offering the services. The information covers names, addresses, contacts, etc. The 'White Pages' includes industrial categories based on standard taxonomies. The 'Green Pages' describes the interface to the service in enough detail for users to write an application to use the web services.

Meanwhile, the UDDI directory provides several ways to let users search for the services they need to build their own applications. For instance, the search can be based on a specified geographic location or on business of a specified type. The UDDI directory will present information, contacts, links and technical data of each service to allow users to evaluate and choose from. Needless to say, such a function makes the search more flexible and efficient.

- SOAP

The SOAP lets a program working in one operating system communicate with a program working in another by using HTTP and XML as information-exchange 
mechanisms. It is therefore provides the means for communication between web services and client applications.

Taken them together, while WSDL defines how web services are described, UDDL describes how to find web services, and SOAP describes how to talk to web services. These technologies constitute a set of baseline specifications that provide the foundation for application integration and aggregation.

\section{Dynamic e-government business models}

Business model is an overall architecture reflecting the core business of an organization. Unlike the traditional governments, an e-government takes on a new look with its framework and operation mode more suitable for the contemporary era [1]. Since e-commerce and e-government are the two comprehensive guiding visions of e-business, e-government is akin to e-commerce in many aspects of business model. In efforts to use information and communication technologies for the civil and political conduct of government, many countries have begun supporting egovernment initiatives. The ultimate goal is to improve government-citizen interactions through an infrastructure built around the "life experience" of citizens $[5]$.

Based on the entities involved, e-government can be classified as G2C, G2B, G2G and G2E $[6,7]$.

Based on its scope, e-government's architecture involves public network access to facilitate government-citizen interaction, intranets to support intra-governmental processes and extranets to support interactions between government and nongovernment organizations [7]. Figure 2 illustrates a general architectural model for egovernment.

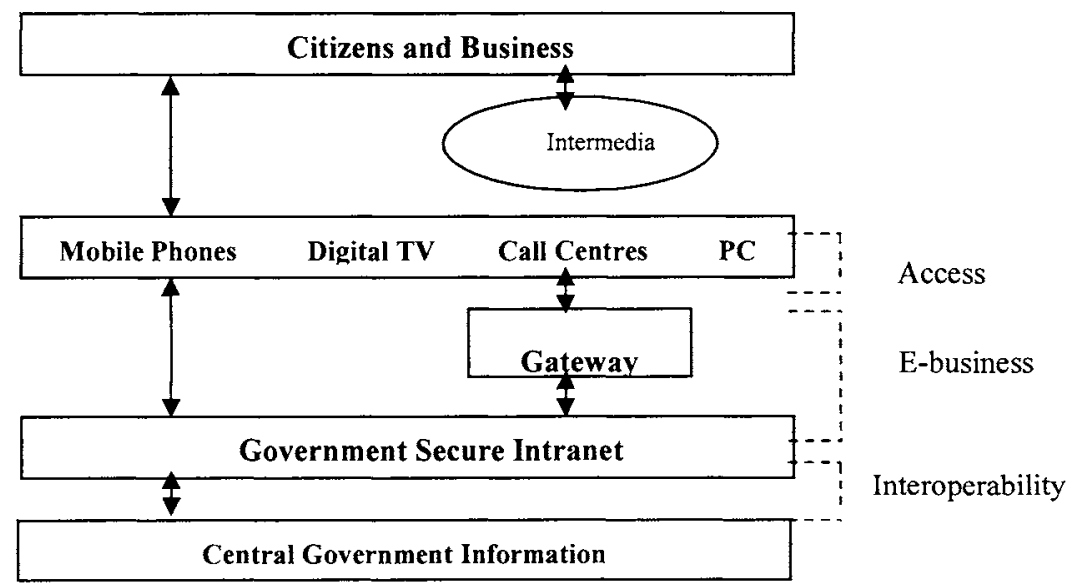

Fig. 2. Architectural model of dynamic e-government 


\section{Merits of web services to dynamic e-government}

Web services produce significant benefits, mainly involving interoperability, flexibility, automatic integration and cost-effectiveness.

- Interoperability

As presented, the significant feature of web services is language-, platform- and location-independent. It provides a means for different organizations with different applications and platform to conduct dynamic e-government across a network, and in turn offering a new range of possibilities for organizations and their partners to develop business solutions.

- Flexibility

Using the traditional approaches to build business applications like in-house custom development software solution and electronic data interchange (EDI) software mentioned above, companies had to know the information such as who was using the applications, how, when, where and why. With WS, companies can build applications without having to know this information. More importantly, each company can have choices of becoming a provider or consumer of services. New revenue streams and new business models would be generated by using existing software assets and integrating them with other businesses.

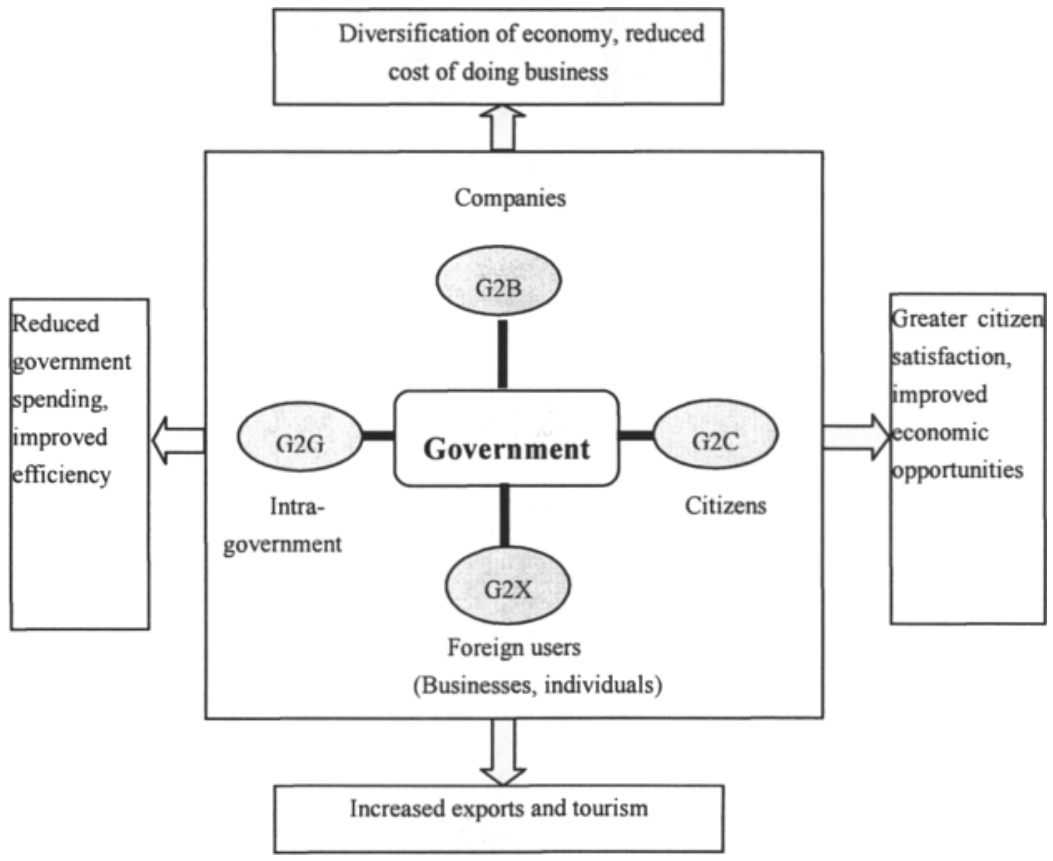

Fig.3. Benefits of dynamic e-government to key stakeholders

- Automatic integration 
Since applications are written to the new standards, WS enable the applications to be automatically integrated regardless where they originate. This provides large potential benefits to the achievement of dynamic e-government.

- Cost-effectiveness

The flexibility of WS also benefits companies to switch to a different preferred supplier more swiftly and smoothly than has ever been possible without disrupting processes or requiring new investments. For this reason, web services are costeffective.

From the standpoint of key stakeholders, the benefits generated by dynamic egovernment are displayed in the Figure 3.

\section{Challenges}

E-government is facing a number of exceptional challenges because of its strict and commitment natures of relationships of $\mathrm{G} 2 \mathrm{C}, \mathrm{G} 2 \mathrm{G}, \mathrm{G} 2 \mathrm{~B}$ and $\mathrm{G} 2 \mathrm{E}[4,8]$. In spite of the considerable literature on e-government, we still do not have a comprehensive, holistic framework in place that assesses the potential of e-government by taking into consideration all the critical dimensions [4]. The major technological challenges include two aspects [9].

\subsection{Equality of access}

Productive, meaningful e-government means citizens must be able to access critical information and services through a variety of devices at their convenience. However, there is an obvious risk that government services and democratic processes will be more accessible to some than to others due to the problem of 'digital divide' of different kinds, between rich and poor countries or regions, between groups within one country differing on social or economic status, age, education level or cultural background and between urban and rural areas [7].

From managerial perspective, to mitigate the digital divide at local or national level is relatively easy to achieve. However, to achieve this goal at international level is a hardship task. Many issues, such as democracy, national development, international aid, local and international business, as well as binational and multinational cooperation program are on the table. With no intention to discuss the social policies that the government should boost to overcome the risk of digital divide, this paper introduces so-called 'Avatars' technology, which can diminish the digital divide to some degrees.

Avatars are animated humanlike characters in knowledge-based presentation systems. They are intelligent interface agents with varying degrees of conversational ability. Technically, they have modules for language processing, interaction management, manipulation interface output animation and so on. Avatars can be helpful in diminishing digital divide because users do not need to be trained in usage of the communication protocols. Every citizen has the skill to use them. 


\subsection{Security and privacy}

The issues of security and privacy in e-government are vital to maintain the public trusts. Old government systems were difficuit to access and often provided little in the way of useable data. But they were usually very secure. Only a handful of individuals could access them and security procedures were relatively easy to set and carry out. This situation is not so with web-based e-government systems. Given the reason that the technical solutions to security in e-commerce are transferable to and applicable in e-government, this section focuses on privacy issue.

Privacy is defined as 'the right of individuals to control the collection, use and dissemination of their person information that is held by others'. Also, it is widely understood as the ethical obligations associated with the collection and use of personal information. Its principles include transparency, fairness, access and security. However, recent surveys have found that while small percentage of government entities have accepted the practices for wide spread use, the evolving technologies (such as click streams, web bugs and cookies) have created a dramatic increase in the collection, use and movement of information and have in turn eroded the perception of personal privacy [4]. Thus it is not surprising that consumers and citizens rank the lack of privacy protection as the foremost deterrent for conducting online transactions.

In addition, some privacy experts contend that a privacy divide, much like the digital divide is developing. The contention is that lower income households have to face more risk of privacy exposure than the middle and upper income households, because they often have no privacy choice when receiving benefits from government or when they attain free services from a company. Certainly privacy divide is not directly related to e-government. However, if this issue does not well addressed, it will effect e-government development in the future.

Developing appropriate security and privacy policies while balance the demands of open public access is a great challenge. The 'holistic view' approach is often recommended, in which security and privacy requirements have to be specified and implemented for each lay of the strategy, process, interaction and data and information.

\section{Conclusion}

Nowadays, a majority of the world's government has Web sites that offer citizens basic information about government organizations, processes and programs, and the quality of these sites is improving at a rapid pace. Although for obvious reasons, egovernment within the most economically developed nations (such as the US, UK, EU, Canada and Australia) has progressed faster than within developing countries, developing countries have started to apply new technologies to build their egovernments. At present, e-government construction is regarded as one of the most important tasks for the national economy and society informatization in China [1]. In our country, strategies and initiatives of e-government are underway. The national EGovernment project is currently undertaking by the State Information Center [1]. 
With its maturity of technologies and management, e-government is moving towards and will eventually enter into the time of 'one-stop' services. Among others, the technology of Web service will be the major contributor to this achievement [11].

In conclusion, e-government gives rise to new kind of government model, which offers a remarkable set of opportunities to increase the transparency of government operations, improve access to government services and enhance efficiency of government administration [4]. Nevertheless, e-government is still in its evolution and is facing various technical and managerial challenges. It needs to be realized that success of e-government relies on three fundamental factors: business, technology and human.

\section{References}

1. J. J. Ning, "E-government in China: current status, strategies and practice, E-Commerce Technology for Dynamic E-Business", IEEE International Conference(2004).

2. P. Mao, E-Government Guide, Peking University Press, Beijing (2003).

3. B. Medjahed, A. Rezgui, A. Bouguettaya and M. Ouzzani, "Infrastructure for e-government Web services", Internet Computing, IEEE, V 7, Il, 58-65 (2003).

4. M. A. Usman, M. Nadeem, M. Z. A. Ansari, and S. Raza, "Multi-agent Based Semantic Egovernment Web Service Architecture Using Extended WSDL", Web Intelligence and International Agent Technology Workshops, WI-1AT 2006 Workshops. 2006 IEEE/WIC/ACM International Conference on Dec. $2006,599-604$.

5. B. Medjahed, A. Rezgui, A. Bouguettaya.and M. Ouzzani, "Infrastructure for e-government Web services", Internet Computing, IEEE, Volume 7, Issue 1, 58 -65(2003).

6. M. Ramaswamy, and A. N. Selian, "e-Government in Transition Countries: Prospects and Challenges System Sciences", HICSS, Annual Hawaii International Conference (Jan. 2007), 92(2006).

7. P. Salhofer and D. Ferbas, "A Business Process Engine Based E-Government Platform Internet and Web Applications and Services", ICIW' 07 . Second International Conference on 13-19, 54[2007-5].

8. H. L. Liu, Z. J. Zhang and Z. F. Peng, E-Government System Outline, Posts\&Telecom Press, Beijing (2005).

9. X. N. Su Xinning, E-Government Technologies, National Defense Industry Press (2003).

10. X. B. Ma and D.Y.Ye, Software Platform and Middleware Technologies, Xiamen University Press (2005).

1I. B. Meneklis, A. Kaliontzoglou, C. Douligeris and D. Polemi, "Engineering and technology aspects of an e-government architecture based on Web services", Third IEEE European Conference, 2[2005-12]. 